\title{
Les OTEX, un indicateur de la spécialisation économique des exploitations viticoles françaises
}

\author{
François Legouy \\ Université d'Orléans, Département de Géographie, 10 rue de Tours, BP. 46527, 45065 Orléans Cedex 2, France
}

\begin{abstract}
Résumé. Les OTEX ou orientations technico-économiques sont un indicateur existant dans le recensement de l'agriculture depuis 1979 en France. Cet indicateur découle d'un calcul lié aux revenus des exploitations. Il explique la spécialisation des exploitations agricoles en fonction des revenus attachés à chaque production agricole de chaque exploitation. Il a donné lieu à des représentations cartographiques par les géographes à partir de 1984. Il est à l'origine du concept de modèle agricole ou de système spatialisé agricole, à l'intérieur desquels, les systèmes viticoles ont une place importante. L'objectif de cette analyse est de comprendre comment s'intègrent les communes viticoles dans l'ensemble de la production agricole française, de dresser une typologie des exploitations agricoles dans leur ensemble en mettant l'accent notamment sur les exploitations viticoles à l'aide des OTEX en 2000 comparées à celles de 2010.
\end{abstract}

\section{Introduction}

Les OTEX sont un indicateur précieux pour comprendre la spécialisation économique des exploitations agricoles. Cet indicateur était calculé jusqu'au recensement de 2000 sur la marge brut standard des exploitations. « La marge brute d'une exploitation était alors obtenue en appliquant à chaque hectare de culture (ou de jachère) et à chaque animal un coefficient, dit « coefficient MBS $»^{1}$, puis en sommant ces MBS partielles ». Ce calcul revient à estimer les revenus moyens de l'exploitation en relation avec les surfaces et le cheptel vivant. L'intérêt est qu'il est comparable aux exploitations de l'Union européenne dans la mesure où il est exprimé également en unité de dimension européenne (UDE) ou encore en « équivalent-hectare de blé ». Lorsque plus des deux tiers des revenus d'une exploitation proviennent d'une production particulière, cette dernière correspond à la spécialisation de l'exploitation. Si ce n'est pas le cas, l'exploitation est classée en polyculture et/ou en polyélevage. Cela signifie qu'elle est orientée sur au moins deux spécialités, ce qui ne signifie pas pour autant et nécessairement qu'elle pratique une grande diversité de cultures et/ou d'élevages. Les termes de polyculture et de polyélevage ne doivent donc pas être pris dans leur ancienne signification.

Or, depuis la réforme de la PAC en 2003, en relation avec l'accord du Luxembourg, qui a institué le découplage des aides liées à la production, la marge brute standard a été remplacée par la production brute standard. Cette dernière s'applique au recensement de 2010 pour l'ensemble des pays de l'Union européenne. La production

${ }^{1}$ INSEE, Définitions et méthodes, http://www.insee. $\mathrm{fr} / \mathrm{fr} / \mathrm{methodes} /$ default . asp? page=definitions $/ \mathrm{mar}$ ge-brute-standard.htm brute standard est proche de la MBS, dans le sens où elle « décrit un potentiel de production des exploitations et permet de classer les exploitations selon leur dimension économique en «moyennes et grandes exploitations »ou 《grandes exploitations $»^{2}$. Ainsi, chaque surface utilisée dans la SAU de l'exploitation sert à calculer une valeur moyenne selon un coefficient issu de valeurs moyennes calculées sur une période de plusieurs années. Mais, il faut préciser que ce calcul doit être plus considéré comme un ordre de grandeur que comme une réalité intangible à chaque exploitation. Il représente également une sorte d'échelle relative entre les productions agricoles les unes par rapport aux autres, puisque, par définition, il est établi sur une moyenne de plusieurs années.

« Les différences principales entre les deux concepts [MBSetPBS] sont la méthode de calcul (les paiements directs et les coûts sont exclus du calcul de la PS) $\gg^{3}$.

L'objectif de ce travail est de dresser une typologie et une cartographie des exploitations françaises en comparant les deux recensements des années 2000 et en 2010 pour mieux comprendre l'évolution de la spécialisation des exploitations viticoles.

Le principe de la classification des exploitations agricoles est relativement ancien en géographie et découle de la volonté de dresser des types d'espaces, combinaisons de plusieurs indicateurs préalablement déterminées selon l'analyse choisie, donc de régionaliser, qui est une des finalités de la géographie [1]. C'est ainsi qu'en 1971, et sous la direction de Jacqueline Bonnamour, une typologie

\footnotetext{
${ }^{2}$ INSEE, Définitions et méthodes, http://www.insee. $\mathrm{fr} / \mathrm{fr} / \mathrm{methodes} /$ default . asp? page=definitions/pro duction-brute-standard.htm

${ }^{3}$ http://ec.europa.eu/agriculture/rica/methodolo gy1_fr.cfm
} 
a été réalisée à partir de plusieurs indicateurs statistiques, mais à l'échelle des grandes régions agricoles [2]. Plus près de nous, la typologie utilisée par les géographes ruralistes de l'université de Paris I en 1984 à l'échelle des cantons a été représentée dans un fascicule à l'occasion de la rencontre de l'UGI à Paris en 1984 [3].

Cette méthodologie a été réemployée par F. Auriac et V. Rey, pour la publication de l'atlas de la France rurale en 1998 à la même échelle cantonale pour le deux recensements de 1979 et 1988 [4].

Depuis le recensement de 2000, les services du SCEES ont cartographié les Orientations technico-économiques de la commune en 2000, à une échelle encore plus fine, mais en dégageant seulement 6 classes, et sans préciser autrement les conditions de production de cette carte [5].

Dans notre travail, la typologie est effectuée à l'aide d'une analyse factorielle des correspondances (AFC) suivie d'une classification ascendante hiérarchique (CAH) avec le logiciel Philcarto créé par le géographe Philippe Waniez (Université de Bordeaux IV) qui présente l'énorme avantage d'être gratuit, facile d'accès et efficace : il permet à la fois le calcul multivariée et la réalisation de la cartographie sans autre manipulation fastidieuse. Même si le calcul des OTEX entre 2000 et 2010 a été modifié, il n'en reste pas moins que la comparaison demeure des plus intéressantes. Elle permet de vérifier la pérennité du principe et de la pertinence de cette typologie. L'étude fonctionnera à deux échelles complémentaires.

Dans quelle mesure l'analyse multivariée des OTEX apporte-t-elle des informations déterminantes comparées à l'étude des seules exploitations viticoles?

Quelles sont les évolutions majeures entre les deux recensements de 2000 et de 2010 ?

Un premier bilan à l'échelle de la France entière permettra de resituer les exploitations viticoles par rapport à l'ensemble des exploitations agricoles et leur évolution sur une période de 10 ans.

L'échelle communale sera l'objet d'une analyse statistique multivariée telle que décrite plus haut et déterminera la deuxième partie de l'article.

\section{Le bilan viticole français à l'échelle nationale et à l'aune des OTEX}

A l'échelle de la France, il est particulièrement instructif de considérer l'évolution des exploitations viticoles dans l'ensemble des exploitations agricoles à l'aune des OTEX. Nous avons déjà réalisé un premier bilan de l'évolution des surfaces viticoles à plusieurs échelles et pas de temps dans un précédent article [6], mais qui n'était pas basé sur le calcul des OTEX. Nous n'y reviendrons pas, mais nous pouvons dans un premier temps comparer la place des exploitations viticoles dans l'ensemble des exploitations en dehors de la spécialisation des OTEX (cf. Tableau 1).

\subsection{Une place modeste des exploitations viticoles françaises pour des tailles réduites selon le recensement de l'agriculture en $\mathbf{2 0 0 0}$}

Le nombre des exploitations viticoles correspond environ au cinquième du total des exploitations agricoles. Ce
Tableau 1. La place des exploitations viticoles dans l'ensemble des exploitations agricoles françaises (2000).

\begin{tabular}{|c|c|c|c|c|}
\hline $\begin{array}{c}\text { Exploitations } \\
\text { agricoles }\end{array}$ & Nbre expl & Nbre expl (\%) & SAU (ha) & SAU (\%) \\
\hline $\begin{array}{c}\text { Total } \\
\text { exploitations }\end{array}$ & 663807 & 100 & 27856312,89 & 100 \\
\hline $\begin{array}{c}\text { Exploitations } \\
\text { viticoles }\end{array}$ & 143747 & 21,7 & 3036610,50 & 10,9 \\
\hline \multicolumn{5}{|l}{ Source : RGA 2000 } \\
\hline
\end{tabular}

Tableau 2. La place des exploitations viticoles dans l'ensemble des exploitations agricoles françaises à travers le prisme des OTEX (2000).

\begin{tabular}{|c|c|c|c|c|}
\hline OTEX & $\begin{array}{c}\text { Nbre } \\
\text { exploitations }\end{array}$ & SAU & $\begin{array}{c}\text { Nbre } \\
\text { exploitations \% }\end{array}$ & SAU \% \\
\hline $\begin{array}{c}\text { Cultures } \\
\text { céréalières }\end{array}$ & 134391 & 9561083 & 20,2 & 34,3 \\
\hline $\begin{array}{c}\text { Cultures } \\
\text { maraîchères et } \\
\text { fruitières, } \\
\text { horticulture }\end{array}$ & 41089 & 408040 & 6,2 & 1,5 \\
\hline $\begin{array}{c}\text { Elevage } \\
\text { polyélevage }\end{array}$ & 32983 & 964018 & 5,0 & 3,5 \\
\hline $\begin{array}{c}\text { Viticulture } \\
\text { d'appellation }\end{array}$ & 59469 & 705485 & 9,0 & 2,5 \\
\hline $\begin{array}{c}\text { Autre } \\
\text { viticulture }\end{array}$ & 32835 & 396887 & 4,9 & 1,4 \\
\hline Total viticulture & 92304 & 1102372 & 13,9 & 4,0 \\
\hline $\begin{array}{c}\text { Association } \\
\text { production } \\
\text { végétale et } \\
\text { animale }\end{array}$ & 45525 & 3655443 & 6,9 & 13,1 \\
\hline Polyculture & 31220 & 838854 & 4,7 & 3,0 \\
\hline $\begin{array}{c}\text { Autres } \\
\text { associations }\end{array}$ & 25237 & 447933 & 3,8 & 1,6 \\
\hline S-Total & 663041 & 27854926 & 99,9 & 100,0 \\
\hline Sources : RGA 2000 & 10877183 & 39,2 & 39,0 \\
\hline
\end{tabular}

chiffre est important au regard des surfaces viticoles en jeu. De fait, il existe un net déséquilibre entre le nombre des exploitations et les surfaces viticoles, deux fois moindres que le nombre des exploitations en valeur relative. Cette faiblesse montre l'importance des exploitations détenant de petites surfaces au moins pour certaines d'entre elles.

L'analyse des OTEX en 2000 (cf. Tableau 2) conforte et accentue la première impression. Comparé au premier tableau, la place relative des exploitations est plus réduite et celle des surfaces viticoles davantage encore. Il manque environ $36 \%$ des exploitations viticoles (soit $(92.304 / 143.747) \times 100$ ) et $64 \%$ (soit $(1.102 .372 / 3.036 .610) \times 100)$ des surfaces viticoles. Ce différentiel comparé à l'ensemble des exploitations sans tenir compte des spécialisations exprime en creux l'importance des exploitations détenant des surfaces viticoles mais dont la spécialisation est dans une autre production agricole. La viticulture présente donc une relative ubiquité dans le concert de l'agriculture française. Cette ubiquité se retrouve dans le différentiel spatial entre les communes viticoles au sens large, c'est-à-dire celles dont au moins une exploitation travaille des vignes dans la commune du siège d'exploitation ou à proximité 


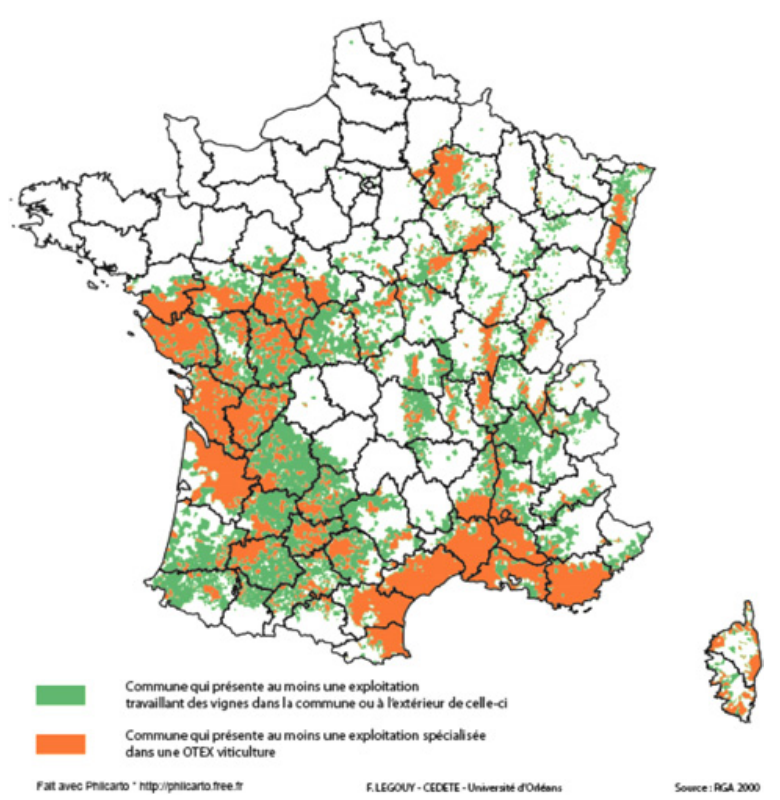

Figure 1. Le différentiel entre les communes viticoles et les communes spécialisées dans une OTEX viticulture (2000).

et les communes viticoles qui possèdent au moins une exploitation agricole spécialisée (répertoriée en OTEX) dans la viticulture. (cf. Fig. 1).

La répartition des communes viticoles est beaucoup plus large que celle des seules communes spécialisées dans la viticulture (OTEX viticulture). Ceci est particulièrement vérifié dans la moitié occidentale de la France qui est connue pour avoir été pendant longtemps la France la plus rurale et traditionnelle, la moins industrialisée. La similitude est plus importante dans le Sud-Est méditerranéen où d'une manière générale la place de la vigne est très prégnante liée aux racines historiques de la viticulture française ainsi qu'en Champagne, en Bourgogne et dans le Bordelais en raison de la réputation et des revenus élevés tirés de la production et de la commercialisation du vin.

L'analyse plus détaillée du Tableau 2 montre la faiblesse dans la spécialisation des exploitations et des surfaces viticoles par rapport à l'ensemble des exploitations spécialisées : $14 \%$ des exploitations pour $4 \%$ des surfaces concernées, ce qui est très peu. Cette faiblesse rappelle à quel point la viticulture est une économie proche du jardinage qui pendant longtemps a été une production agricole nécessitant des bras et de nos jours qui se contente encore de petites exploitations. Il n'est point nécessaire dans le cadre d'une certaine viticulture d'appellation de détenir beaucoup de vignes pour gagner dignement sa vie. ${ }^{4}$ Le différentiel des pourcentages entre le nombre des exploitations et les surfaces est également symptomatique d'un héritage foncier marqué par l'émiettement des surfaces, la faiblesse des remembrements, marqué plus qu'ailleurs excepté pour les exploitations spécialisées dans les cultures fruitières, horticoles et maraîchères.

${ }^{4}$ Pour reprendre le témoignage d'un vigneron de VosneRomanée en Bourgogne : «On vit très bien à Vosne avec seulement 6 ha de vignes $»$.
Tableau 3. La place des exploitations viticoles dans l'ensemble des exploitations agricoles françaises (2010).

\begin{tabular}{|l|c|c|c|c|}
\hline $\begin{array}{c}\text { Exploitations } \\
\text { agricoles }\end{array}$ & Nbre expl & Nbre expl (\%) & SAU (ha) & SAU (\%) \\
\hline $\begin{array}{c}\text { Total } \\
\text { exploitations }\end{array}$ & 489977 & 100 & 26963251,56 & 100 \\
\hline $\begin{array}{c}\text { Exploitations } \\
\text { viticoles }\end{array}$ & 82051 & 16,7 & 788633,00 & 2,9 \\
\hline \multicolumn{5}{|l}{ Source : RGA 2010 } \\
\hline
\end{tabular}

L'ensemble des OTEX à l'échelle nationale démontre encore une concentration foncière importante pour la production végétale, hormis la viticulture, et pour la production animale qui, sans compter une combinaison possible et réelle entre les deux catégories, totalisent plus des trois quart des exploitations et plus de $80 \%$ des surfaces.

Le système polycultural et polyélevage dont nous avons rappelé les limites sémantiques dans l'introduction constitue cependant l'héritage d'une antique agriculture où « tous les oufs n'étaient pas mis dans le même panier ». Il faut entendre par cette expression le fait que les productions agricoles étaient volontairement diversifiées afin de limiter les effets d'une «mauvaise année » dans une production donnée. Cette poly-production correspondait également à une complémentarité des terroirs où, pour simplifier le schéma, les plateaux étaient dévolus à la production céréalière, les fonds de vallées aux prairies pour le pacage des animaux et les versants de vallées ou liés à des talus entre plateaux et plaines étaient cultivées avec des vignes et des arbres fruitiers. Cette complémentarité a largement disparu avec la révolution des transports qui a renforcé la spécialisation régionale de l'agriculture. Effectivement, il n'était plus nécessaire de produire de tout, partout. La révolution agricole qui s'est diffusée largement après la seconde guerre mondiale a encore renforcé le processus. Les champs les plus faciles d'accès sur les surfaces planes ont été privilégiés, tandis que les versants trop pentus pour les tracteurs ont été délaissés et se sont couverts peu à peu de friches. La mécanisation a encore eu comme conséquence la banalisation des paysages agraires, avec un agrandissement des parcelles afin d'optimiser l'utilisation des tracteurs. Il n'en demeure pas moins que la polyculture et le polyélevage continuent d'exister, dans une certaine mesure...

La comparaison avec les chiffres de 2010 renforce cette idée de spécialisation de l'agriculture (cf. Tableaux 3 et 4).

\subsection{La viticulture française en 2010 : un renforcement de la concentration des terres par exploitation}

En 2010, en comparaison avec le RGA de 2000, le nombre d'exploitations travaillant des vignes est en net recul de plus de $40 \%$ et les surfaces viticoles de plus de $70 \%$. Dans le même ordre d'idée, la place relative de la viticulture a sensiblement reculé que ce soit en nombre d'exploitations ou en surfaces viticoles. On pouvait penser a priori que la viticulture aurait mieux tenu face à la crise profonde qui la traverse depuis le début des années 2000. C'était oublier un peu vite que nombre d'exploitations et de surfaces viticoles 
Tableau 4. La place des exploitations viticoles dans l'ensemble des exploitations agricoles françaises à travers le prisme des OTEX (2010)_L'évolution entre 2000 et 2010.

\begin{tabular}{|c|c|c|c|c|c|c|}
\hline \multicolumn{2}{|c|}{ La spécialisation des exploitations exprimée par les OTEX (2010) } & \multicolumn{2}{|c|}{ Evolution 2000-2010\% } \\
\hline & $\begin{array}{c}\text { Nbre } \\
\text { exploitations }\end{array}$ & SAU & $\begin{array}{c}\text { Nbre } \\
\text { exploitations } \\
\%\end{array}$ & SAU \% & Nbre expl \% & SAU \% \\
\hline $\begin{array}{c}\text { Cultures } \\
\text { céréalières }\end{array}$ & 118763 & 9181899 & 24,2 & 34,1 & $-11,6$ & $-4,0$ \\
\hline $\begin{array}{c}\text { Cultures } \\
\text { maraîchères } \\
\text { et fruitières, } \\
\text { horticulture }\end{array}$ & 33167 & 410349 & 6,8 & 1,5 & $-19,3$ & 0,6 \\
\hline Elevage & 197852 & 11510909 & 40,4 & 42,7 & $-24,0$ & 5,8 \\
\hline polyélevage & 13572 & 876642 & 2,8 & 3,3 & $-58,9$ & $-9,1$ \\
\hline $\begin{array}{c}\text { Viticulture } \\
\text { d'appellation }\end{array}$ & 61765 & 892501 & 12,6 & 3,3 & 3,9 & 26,5 \\
\hline $\begin{array}{c}\text { Autre } \\
\text { viticulture }\end{array}$ & 8190 & 203432 & 1,7 & 0,8 & $-75,1$ & $-48,7$ \\
\hline $\begin{array}{c}\text { Total } \\
\text { viticulture }\end{array}$ & 69955 & 1095933 & 14,3 & 4,1 & $-24,2$ & $-0,6$ \\
\hline $\begin{array}{c}\text { Association } \\
\text { production } \\
\text { végétale et } \\
\text { animale }\end{array}$ & 35816 & 3301588 & 7,3 & 12,2 & $-21,3$ & $-9,7$ \\
\hline Polyculture & 10051 & 512579 & 2,1 & 1,9 & $-67,8$ & $-38,9$ \\
\hline $\begin{array}{c}\text { Autres } \\
\text { associations }\end{array}$ & 1854 & 17467 & 0,4 & 0,1 & $-92,7$ & $-96,1$ \\
\hline \begin{tabular}{c} 
S-Total \\
\hline
\end{tabular} & 481030 & 26907366 & 98,2 & 100 & $-27,5$ & $-3,4$ \\
\hline
\end{tabular}

correspondaient à des reliquats d'une viticulture basée sur l'autoconsommation familiale et/ou d'une viticulture de consommation courante, de vin de table, hors appellation digne de ce nom, donc peu rentable à la commercialisation. Surtout, la consommation de vin a fortement chuté comme le nombre de consommateurs traditionnels : ouvriers et paysans.

Cette viticulture a été largement responsable de la surproduction de la première moitié du $\mathrm{XX}^{\mathrm{e}}$ siècle, basée en partie sur des hybrides et producteurs directs. Elle s'explique par plusieurs facteurs, en particulier par la volonté des petits paysans, avant et au sortir de la Grande guerre, de cultiver eux-mêmes leurs vignes. La multiplication des hybrides demandant peu de soins et produisant avec de gros rendements y a fortement contribué. Le gouvernement français a programmé sa disparition avec les différentes lois amenant à la création des AOC et le « statut de la viticulture » qui s'est échelonné au cours des années 1930 et 1950. Cette viticulture ne cesse de disparaître depuis plus d'un siècle (F. Legouy, 2014). Elle a été souvent ignorée des analyses, car de moins en moins perceptible de recensements en recensements de l'agriculture et le plus souvent inconnue des enquête annuelles.

Elle a quasi disparu au profit d'une viticulture de qualité et davantage spécialisée, rétractée et visible dans les OTEX viticultures (cf. Fig. 2). En effet, le nombre de communes viticoles sans spécialisation a fortement régressé entre 2000 et 2010 dans l'ensemble de la France et plus particulièrement dans le Nord-Est et le SudEst du pays. À cet égard, la transformation des régions méditerranéennes résulte d'une profonde restructuration

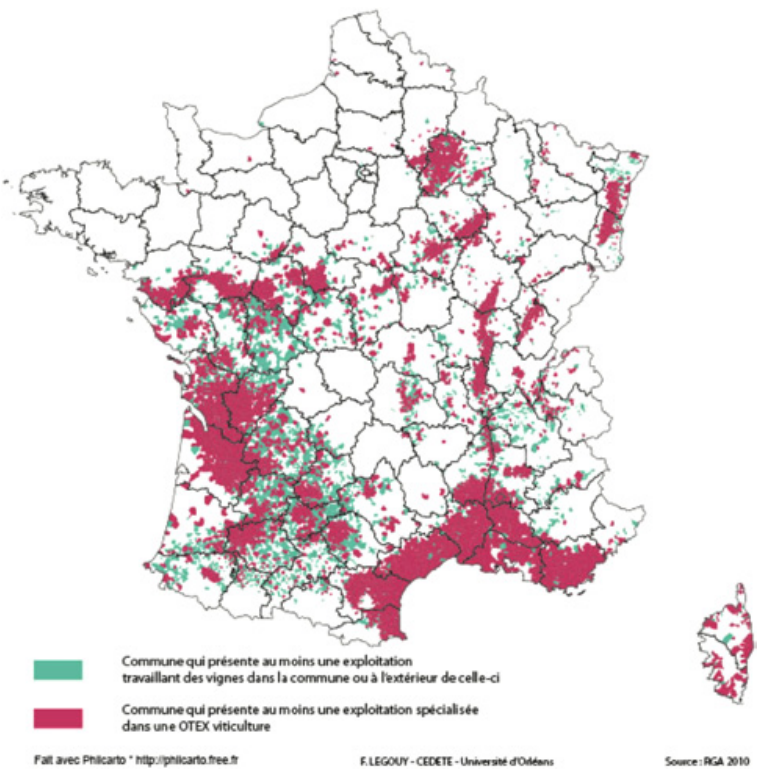

Figure 2. Le différentiel entre les communes viticoles et les communes spécialisées dans une OTEX viticulture (2010).

de la viticulture au profit des appellations de qualité principalement IGP, mais aussi AOP.

La comparaison entre les deux recensements doit être considérée avec beaucoup de précaution. Le calcul des MBS et des PBS n'étant pas identique, les valeurs relatives sont davantage à prendre en compte et doivent être considérées comme des ordres de grandeur. La place de la viticulture d'appellation a nettement progressé et en nombre d'exploitations et en surfaces viticoles au contraire de la viticulture « autre $»$ qui exclut la production des AOP et des IGP. Cette « autre viticulture »a ainsi perdu plus des trois quart de ses exploitations et près de la moitié de ses surfaces. La viticulture d'appellation est la seule spécialisation agricole à avoir un taux de croissance et des valeurs absolues en progression pour ses exploitations et plus encore pour ses surfaces. Mais, dans le cadre d'un agrandissement des surfaces par exploitation : la taille moyenne des exploitations viticoles spécialisées augmente de 11,8 ha à 14,5 ha sur dix ans comme a augmenté le nombre de salariés dans cette viticulture. Ce gain est d'abord révélateur d'une rentabilité certaine à la production, plus que toute autre production agricole. Il témoigne ensuite d'un effort séculaire en faveur d'une production de qualité en vue d'une commercialisation globalement gratifiante sur le marché national et international, et ce, malgré la baisse de la consommation intérieure et la montée de la concurrence des pays du Nouveau Monde. Elle est enfin le fruit d'une mise en avant et d'une protection patrimoniale et labellisée d'une production territoriale de qualité, maîtrisée sur le plan technique et non délocalisable, avec l'utilisation de cépages de réputation internationale qui sont utilisés de plus en plus dans les nouveaux pays producteurs comme dans les anciens pays viticoles, au détriment des cépages locaux, de plus en plus appelés « cépages modestes ». La France est à cet égard encore un modèle copié à l'envi par tous ses concurrents dont les acteurs viennent se former 

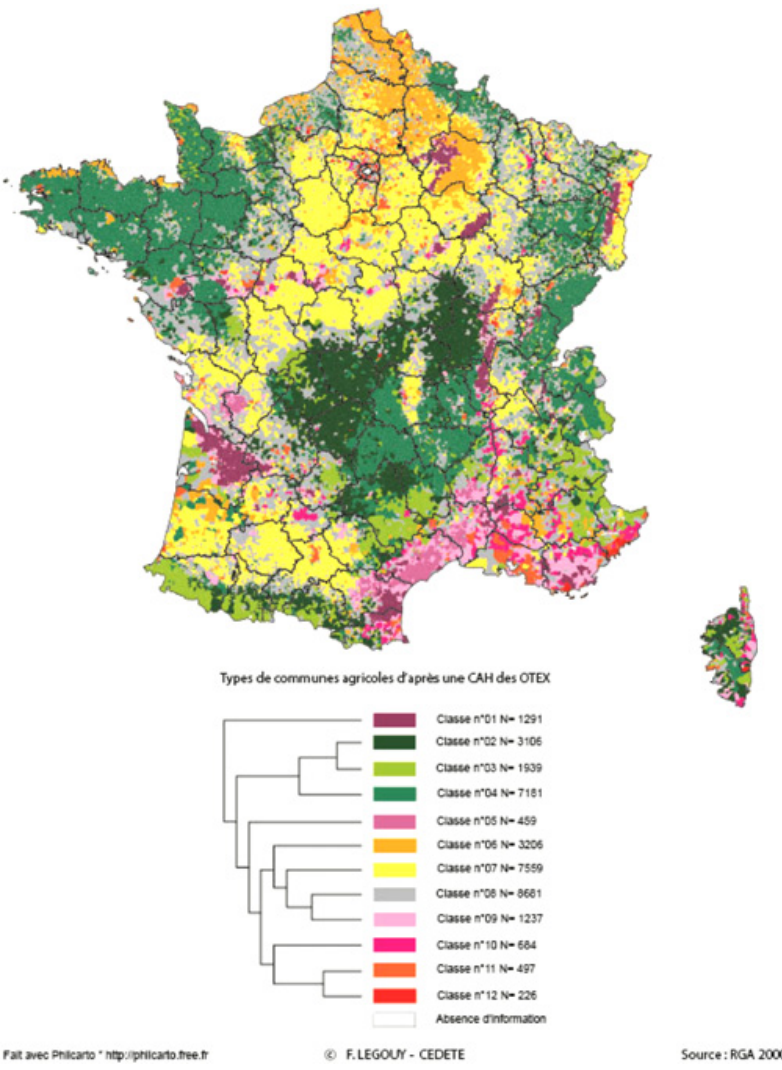

Figure 3. La spécialisation des exploitations viticoles dans l'ensemble des systèmes de productions agricoles français en 2000.

dans les écoles de viticulture et d'œnologie de Montpellier, de Dijon, ou de Bordeaux....

L'analyse de la combinaison des OTEX doit préciser cette spécialisation.

\section{La spécialisation régionale de l'agriculture : une réalité visible avec la cartographie des différents types d'OTEX}

Pour mieux comprendre à la fois comment s'insère spatialement la viticulture française dans l'ensemble de l'agriculture et l'importance de la spécialisation viticole, il n'est pas de meilleur document que celui de la carte des combinaisons des OTEX pour toutes les exploitations agricoles en 2000 et en 2010. Ces deux cartes sont accompagnées par les graphiques des profils de chaque type déterminés par la classification ascendante hiérarchique.

\subsection{Les combinaisons des OTEX en 2000 : une carte qui rappelle le lien unissant l'agriculture et les milieux physiques français}

La première carte a été réalisée à l'aide d'une analyse factorielle des correspondances (AFC) suivie d'une classification ascendante hiérarchique $(\mathrm{CAH})$, avec l'application Philcarto du géographe $\mathrm{Ph}$. Waniez (Bordeaux IV). L'analyse factorielle permet de comprendre dans un
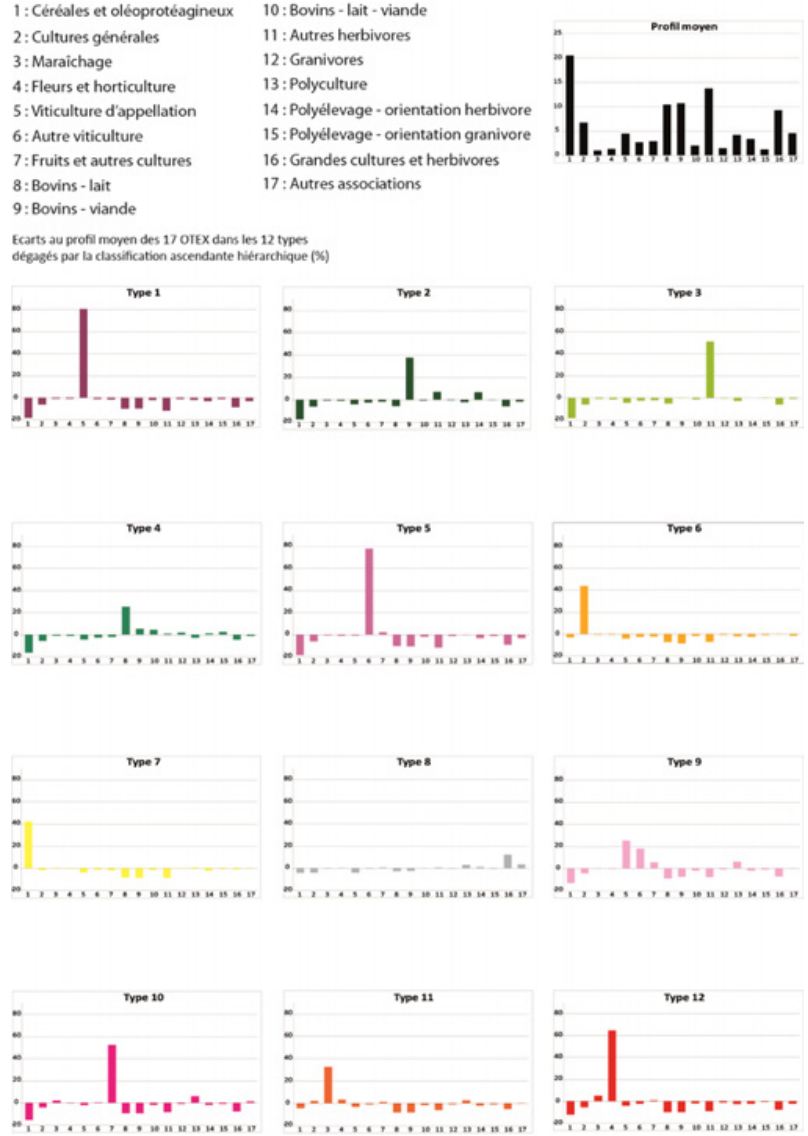

Figure 4. le profil moyen des OTEX pour l'ensemble des exploitations agricoles et le profil de chaque type représenté sur la carte pour toutes les variables en 2000.

premier temps comment les diverses OTEX sont en relation les unes avec les autres et de dresser une typologie de ces combinaisons.

L'analyse a été effectuée à partir du nombre des exploitations agricoles dans chaque commune afin de limiter la prédominance des surfaces dévolues aux céréales et à l'élevage, bien plus importantes que celles liées à la vigne. En définitive trois documents sont réalisés : la carte proprement dite, l'arbre de hiérarchisation des différents types et les graphiques qui positionnent toutes les variables dans chaque type (Fig. 4). Les douze types retenus doivent être compris comme des combinaisons d'OTEX qui ont des comportements statistiques proches les uns des autres. Pour 17 variables à l'origine, il a été retenu 12 types. Ceci explique que certains types ne relèvent que d'une seule variable. C'est en particulier le cas pour les types 1 et 5 qui sont liés à la viticulture d'appellation (type 1) et l' « autre viticulture » (type 5). Le type 9 est une combinaison des deux variables liées à la viticulture et à celle des fruits et autres cultures. Nous l'appellerons 《viticulture mixte ». Sur l'arborescence hiérarchique, il est proche de la classe 8 représentant les grandes cultures et herbivores et dans une moindre mesure le polyélevage. Ce type indique donc une association entre la viticulture sous toutes ses formes et d'autres productions agricoles principales, situation relativement classique dans certains vignobles secondaires et/ou dans des régions où 
la viticulture est en situation marginale et périphérique par rapport à des vignobles renommés. Nous y reviendrons. Il faut retenir également que dans la hiérarchie des types, la viticulture d'appellation se démarque de toutes les autres catégories, en particulier du groupe des cultures céréalières (couleurs jaune et orange) et de l'élevage (couleurs vertes) qui sont elles-mêmes bien à part. Les types 5 et 9 sont davantage mêlés aux autres types.

La carte est très riche d'enseignements. Elle met d'abord en exergue l'influence des milieux naturels sur la production agricole française. On parle souvent d'agriculture dé-territorialisée, hors sol, industrielle [7] :

«L'agriculture tend à se concentrer vers les zones littorales, vers les grandes zones de concentration des communications. La géographie des communications l'emporte sur la géologie et la richesse agronomique des sols, au moins partiellement. [...] Mais la délocalisation opère également à l'intérieur du territoire national : les 10 principaux départements producteurs, tous situés au nord d'une ligne Nantes-Strasbourg, produisent autant que les 45 plus petits. [...] Cette concentration de la production se conjugue avec une forte spécialisation [...] Ce double phénomène de concentration et de spécialisation produit un paysage agricole complètement transformé qui n'a plus rien à voir avec une France à la surface de laquelle on produisait un peu de tout partout. [...] Aux terroirs ont succédé les bassins : bassin céréalier, bassin allaitant, bassin porcin, bassin laitier. [...] Abstraite, hors-sol et mobile : telle se découvre une partie de l'agriculture. Comme tous les autres secteurs économiques et socioprofessionnels, elle est devenue précaire dans sa localisation, de telle sorte qu'il est devenu impossible de la considérer comme un antidote « naturel »au déménagement du territoire. »

Cette carte semble démontrer le contraire à l'échelle des communes, plus exactement elle confirme que toute l'agriculture française n'est pas en voie de délocalisation en direction des littoraux et des grands ports internationaux. Elle ne dit rien, évidemment sur la production et les rendements, encore qu'il soit de notoriété que les grandes cultures, les cultures céréalières et oléo-protéagineuses (variables 1, 2 et 16) sont réalisées avec des rendements très élevés dans le cadre d'une agriculture productiviste. Mais elle tient compte d'une certaine manière des revenus issus de la production et donc des rendements.

Les milieux physiques sont représentés par les grands ensembles morphostructuraux, ${ }^{5}$ particulièrement visibles ici : les massifs anciens tels que le Massif armoricain, le Massif central, les Ardennes, les Vosges sont nettement distinguables. Il en est de même pour les Bassins parisien et aquitain dont les auréoles géologiques d'affleurement sont très bien dessinées avec les classes 6 et 7 . Le Midi méditerranéen et les chaînes de montagne Alpes,

\footnotetext{
${ }^{5}$ Il faut entendre par « grands ensembles morphostructuraux 》 la combinaison géomorphologique entre relief et géologie ; combinaison qui s'est réalisée au cours d'une évolution sur le très long terme entre les roches d'origine sédimentaire et celles d'origine plutonique, les forces tectoniques et les systèmes morphogéniques en relation avec les paléoclimats et climats plus récents qui les ont façonnés [9].
}

Jura, Pyrénées sont également nets. A la limite, il serait presque possible d'affirmer que cette carte est le fidèle reflet pour le territoire français et à petite échelle de la diversité de ses terroirs. Dans ce contexte, la relation entre la production de céréales correspond très clairement aux Bassins sédimentaires alors que l'élevage est lié aux régions de massifs anciens et aux chaînes de montagnes. La spécialisation de cet élevage est répartie entre les régions d'élevage bovin pour la viande (parties septentrionales du Massif central, bordures septentrionales des Pyrénées, nord-ouest et nord-est de l'Aveyron), et pour le lait (Massif armoricain, département des Vosges, Chaîne du Jura, Alpes du Nord, centre du massif central) et les régions d'élevage d'ovins et de caprins (Alpes du Sud, bordures méridionales et occidentales du Massif central, Pyrénées, Corse). Les élevages de granivores sont mêlés à d'autres productions.

La distribution des vignes au contraire est davantage localisée dans les grandes vallées fluviales, leurs vallées affluentes, les sites et les escarpements d'abri par rapport aux vents apportant la pluie et/ou le froid et les régions méditerranéennes. La spécialisation de la viticulture comparée aux autres productions agricoles est donc plus restreinte que sur la carte de la Fig. 1. Il existe comme pour les cartes précédentes un gradient spatial viticole entre la viticulture d'appellation, les autres viticultures et la viticulture mêlée à d'autres productions agricoles. Ce gradient est notamment visible autour des vignobles bordelais, du Languedoc, de la Bourgogne, du Cognaçais par exemple, mais complètement absent de la Champagne, mettant en évidence une spécialisation en vins de qualité, spatiale, unique et précoce pour cette dernière région. $\mathrm{Ce}$ gradient est révélateur d'une spécialisation intense au cœur des vignobles puis décroissante à leurs marges.

Enfin, les cultures maraîchères et fruitières sont proches des grandes agglomérations dans des ceintures auréolaires de plus en plus lointaines et discontinues en fonction de la croissance et de l'extension urbaine et périurbaine. Elles sont également nombreuses dans le SudEst et le Sud-Ouest, dans une certaine proximité de la viticulture.

Un autre point important de la carte est celui de l'existence d'une bande de transition entre les principaux éléments de la typologie des OTEX illustrant les massifs anciens et les bassins sédimentaires ; bande réalisée par la présence de la classe 8 en gris sur la carte qui associe les variables de la polyculture et du polyélevage. Cette bande, qualifiée encore de marge d'indécision dans l'atlas de 1998 [4], est le témoin, certes ténu et partiellement modifié, de l'existence d'un ancien système polyculture-polyélevage qui autrefois était beaucoup plus la règle et qui a disparu avec la spécialisation régionale de l'agriculture.

\subsection{La carte des OTEX en 2010 : une accentuation de la spécialisation des exploitations viticoles}

La comparaison avec l'année 2010 (Fig. 5) vient conforter ce qui a déjà été aperçu sur l'année 2000 : on retrouve sensiblement la même configuration spatiale, mais avec de sérieuses nuances liées à l'évolution de l'agriculture en 


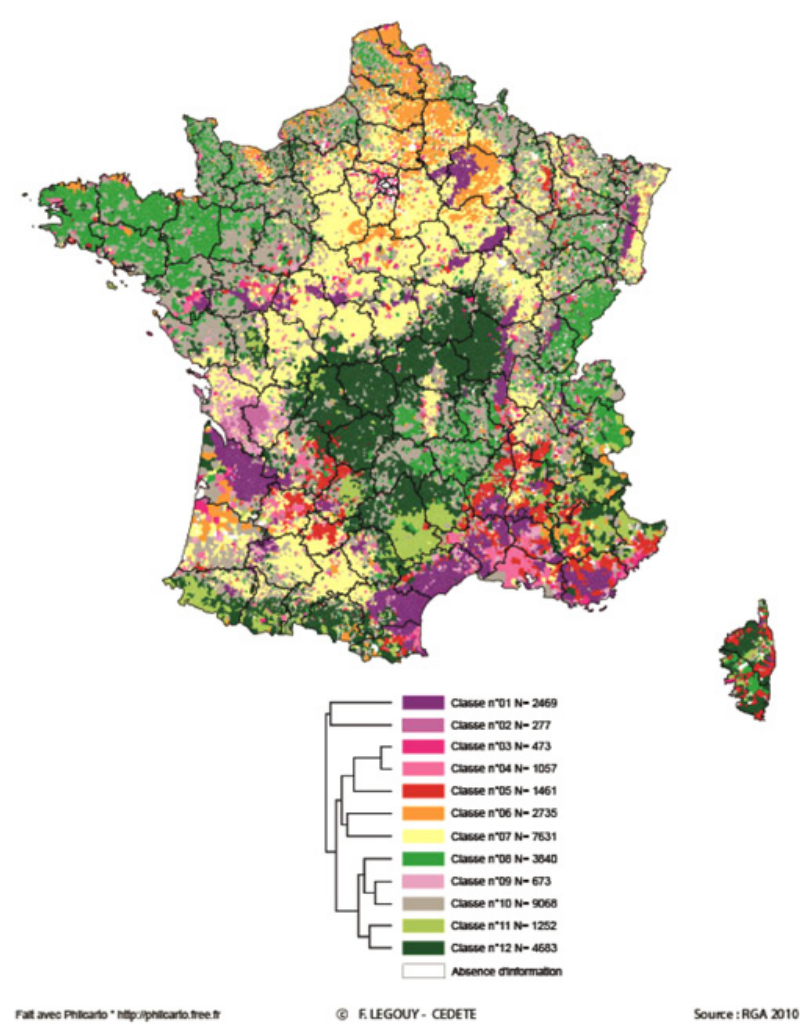

Figure 5. La spécialisation des exploitations viticoles dans l'ensemble des systèmes de productions agricoles français en 2010 .

France et sans doute aussi au nouveau mode de calcul de la production brute standard.

C'est ainsi que les limites spatiales des massifs anciens sont un peu moins nettes. Plus précisément, les cultures céréalières gagnent du terrain au nord du Massif central et le système polyculture-polyélevage empiète sur la bordure orientale du massif armoricain, comme du reste dans le sud du Massif central. De même, l'arbre de la classification met mieux en valeur l'originalité des exploitations viticoles classes 1 et 3 qui se démarquent franchement des autres catégories liées à la production des céréales et de l'élevage, excepté pour la viticulture liée à d'autres productions agricoles (classe 9).

La place de la viticulture a gagné en importance : elle représente désormais $9,6 \%$ du nombre des communes répertoriées pour $8,3 \%$ en 2000 . Surtout, la viticulture d'appellation (classe 1) représente le double 6,9\% de ce qu'elle obtenait $(3,6 \%)$ en 2000 , au détriment de la viticulture mixte et davantage de la 《viticulture autre », en particulier dans les régions du Val de Loire où le trait linéaire est plus net et la région du Languedoc-Roussillon où elle est devenue largement majoritaire.

Cette transformation reflète la restructuration du vignoble languedocien sorti désormais de l'ornière des vins de table qui ne représentent plus en 2010 que $6 \%$ de la production contre $17 \%$ pour les AOP et $77 \%$ pour la production des vins IGP. La viticulture « mixte 》 est encore très présente dans la partie occidentale de la France, plus spécialement dans le Cognaçais, dans la région d'Armagnac et dans la partie aval du Val de Loire,
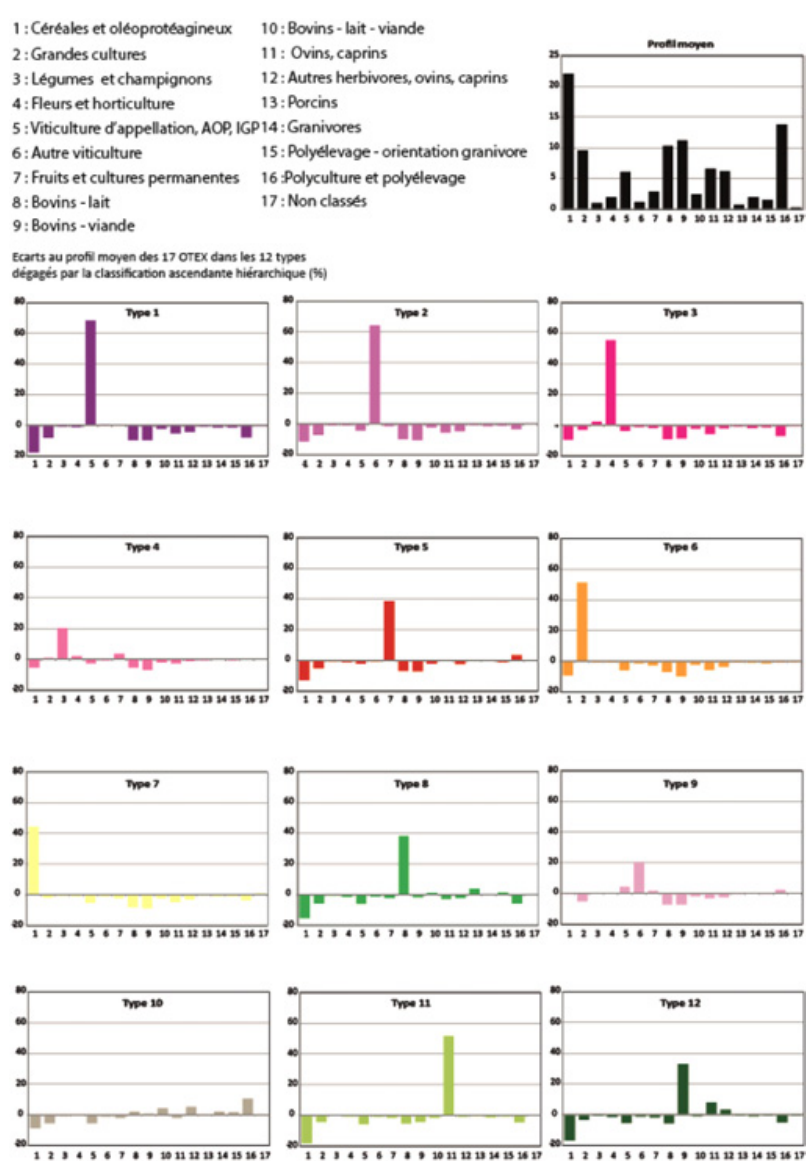

Figure 6. le profil moyen des OTEX pour l'ensemble des exploitations agricoles et le profil de chaque type représenté sur la carte pour toutes les variables en 2010 .

Tableau 5. L'évolution du nombre des exploitations dans les combinaisons d'OTEX par classification ascendante hiérarchique entre 2000 et 2010.

\begin{tabular}{|l|c|c|c|c|}
\hline \multicolumn{5}{|l|}{ les combinaisons d'OTEX dans la classification ascendante hiérarchique : } \\
évolution 2000-2010 \\
\hline $\begin{array}{l}\text { OTEX dominantes issues } \\
\text { des CAH }\end{array}$ & 2000 & $\%$ & 2010 & $\%$ \\
\hline Viticulture d'appellation & 1291 & 3,6 & 2469 & 6,9 \\
\hline viticulture autre & 459 & 1,3 & 277 & 0,8 \\
\hline viticulture mixte & 1237 & 3,4 & 673 & 1,9 \\
\hline horticulture-fruit-légumes & 1407 & 3,9 & 2991 & 8,4 \\
\hline polyculture-polyélevage & 8681 & 24,1 & 9068 & 25,5 \\
\hline cultures céréalières & 10765 & 29,8 & 10366 & 29,1 \\
\hline éegavel & 12225 & 33,9 & 9775 & 27,4 \\
\hline TOTAL & 36065 & 100,0 & 35619 & 100,0 \\
\hline Source : RGA 2000-2010 & \multicolumn{4}{|l}{} \\
\hline
\end{tabular}

beaucoup moins ailleurs. On assiste bien, compte tenu des chiffres en présence et malgré la prudence d'interprétation qui s'impose, à une plus grande spécialisation au profit de la viticulture de qualité représentée par les vins AOP et IGP (classe 1). Les autres combinaisons restent relativement stables, excepté l'élevage qui dans les combinaisons représente un peu plus du tiers des spécialisations présentes sur la carte, contre plus de $27 \%$ en 2000. 


\section{Conclusion}

Les deux analyses réalisées sur les recensements de l'agriculture à 10 ans d'intervalle fournissent des résultats relativement proches. Dans les deux cartes, les grands ensembles physiques apparaissent assez distinctement, davantage en 2000 qu'en 2010. Nous sommes loin d'une agriculture déterritorialisée dénoncée par Bernard Hervieu, ce qui ne signifie pas que ce type d'agriculture n'existe pas en France, mais qu'il n'est pas détectable dans ce genre de statistique.

Le grand intérêt de la carte des combinaisons des OTEX est de positionner les principales agricultures les unes par rapport aux autres et d'en montrer les relations de proximité à la fois statistiques et spatiales. Mais, dans le même temps, elle simplifie la réalité en ne donnant à voir que les exploitations les plus spécialisées dans leurs domaines. C'est donc à la fois une bonne synthèse et une contraction de la réalité qui est la base même de l'exercice de l'analyse multivariée.

La place de la viticulture dans l'espace français se réduit de plus en plus et pour l'essentiel aux vignobles les plus réputés, dans les grandes vallées fluviales, en des sites d'abris par rapport aux vents dominants derrière des escarpements et sur les pourtours méditerranéens, aux principales $\mathrm{AOC}$ et dans certaines régions à des vins de pays (IGP). De ces changements, il découle que le gradient spatial qui était encore nettement visible en 2000, par exemple dans la région du Languedoc-Roussillon comme ailleurs a perdu une partie de sa pertinence en 2010.

Par contre, les deux vignobles de Cognac et d'Armagnac ont conservé leur place dans la typologie en « viticulture autre » correspondant à leurs particularités de produire un vin de faible qualité pour la fabrication d'alcool et de plus en plus des vins IGP à partir de cépages qui sont remis au goût du jour, ugni blanc et colombard.

La spécialisation a gagné de proche en proche toutes les exploitations viticoles en abandonnant une production agricole plus dispersée. Dans ce contexte, La viticulture d'appellation est la seule OTEX qui ne perd pas d'exploitations, mais au contraire gagne à la fois des exploitations et des surfaces, le plus souvent par le passage de certaines exploitations d'une catégorie à l'autre et par la restructuration du vignoble dans son ensemble. Et ce, malgré une rétraction globale du nombre des exploitations agricoles et viticoles.

Si l'ancien président de la $\mathrm{FEVS}^{6}$, Louis-Fabrice Latour, grand négociant bourguignon, regrettait que la France avait perdu la bataille des volumes à l'exportation, il ajoutait également qu'elle dominait et de loin la valorisation des exportations pour ses vins de qualité. Cette constatation pour le marché international corrobore pleinement les évolutions nationales de la viticulture française en l'espace de 10 ans et visibles sur les cartes de cet article.

\section{Références}

[1] Ph. Pinchemel, La Face de la terre, Paris, Armand Colin, 519 p., (1988)

[2] J. Bonnamour, Ch. Gillette, Y. Guermond, « Les systèmes régionaux d'exploitation agricole en France. Méthode d'analyse typologique », Études rurales, $\mathrm{N}^{\circ}$ 43-44, p. 78-169, (1971)

[3] M. Béguin, J.-Cl. Bontron, J.-P. Charvet, V. Rey (dir.), Les exploitations agricoles françaises, Paris, CNRS, (1984)

[4] F. Auriac, V. Rey, 1998, L'espace rural, Collection Atlas de France, Volume 8, Paris, Reclus, La Documentation françaises, p. 56-59, (1998)

[5] Agreste Cahiers n० 3-4, « Recensement agricole $2000 \gg$, p. 29, (2001)

[6] F. Legouy, «La géohistoire de l'espace viticole français sur deux siècles $(1808$ - 2010) : plus ieurs cycles viticoles décryptés.», Espaces Temps. net, Travaux, 17.02.2014, (2014). http://www . espacestemps.net/articles/la-geohistoirede-lespace-viticole-francais-sur-deuxsiecles-1808-2010-plusieurs-cycles-vit icoles-decryptes/

[7] B. Hervieu, J. Viard, Au bonheur des campagnes, La Tour d'Aigues, Editions de l'Aube, 160 p, (2001)

[8] B. Hervieu, Les agriculteurs, Paris, PUF, QSJ ?, $n^{\circ} 3048$, p. 30-37, (1996)

[9] R. Coque, Géomorphologie, Paris, Armand Colin, 430 p., (1977)

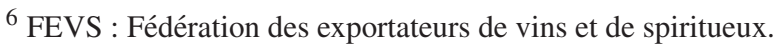

